\title{
Variable exponent trace spaces
}

\author{
by \\ Lars Diening (Freiburg) and Peter Hëstö (Oulu)
}

\begin{abstract}
The trace space of $W^{1, p(\cdot)}\left(\mathbb{R}^{n} \times[0, \infty)\right)$ consists of those functions on $\mathbb{R}^{n}$ that can be extended to functions of $W^{1, p(\cdot)}\left(\mathbb{R}^{n} \times[0, \infty)\right.$ ) (as in the fixed-exponent case). Under the assumption that $p$ is globally log-Hölder continuous, we show that the trace space depends only on the values of $p$ on the boundary. In our main result we show how to define an intrinsic norm for the trace space in terms of a sharp-type operator.
\end{abstract}

1. Introduction. In this article we present a simple approach to trace spaces. Our philosophy is to move away as little as possible from the definition of trace space as consisting of those functions which can be extended to the whole space. The motivation for pursuing this line of investigation is that it provides us with more robust results and methods. We are especially interested in Sobolev spaces with variable exponent. What makes variable exponent spaces stand apart particularly in the current context is that they are not translation invariant, in contrast to their classical counterparts. A glance at the classical approaches (due to Lions, Peetre and others, see, e.g., [3, Section 7], [4, Section 7] and references therein) shows that translation invariance is in many situations at the heart of the matter, starting with the idea that we can define a norm as a Bochner integral of a function from the real line to a Banach space. We believe that our approach can be used also when dealing with other non-translation invariant generalizations of Sobolev spaces, such as other variants of Orlicz-Musielak spaces [32], or spaces with variable smoothness [29].

On an intuitive level we get the variable exponent space by replacing the energy (modular)

2000 Mathematics Subject Classification: 46E30, 46E35.

Key words and phrases: variable exponent, Sobolev space, trace space, intrinsic norm, sharp maximal operator.

L. Diening was supported in part by the Landesstiftung Baden-Württemberg, project Intelligent Materials.

P. Hästö was supported by the Research Council of Norway, project 160192/V30. 


$$
\int_{\Omega}|f(x)|^{p} d x \quad \text { with } \quad \int_{\Omega}|f(x)|^{p(x)} d x,
$$

where $p(x)$ is some function. Exact definitions are given below. Let us review some of the major reasons for why variable exponent spaces have attracted quite a bit of attention lately (see [13] for a bibliography of over a hundred titles on this topic from the last five years). Variable exponent spaces are connected to variational integrals with non-standard growth and coercivity conditions $[2,39]$. These non-standard variational problems are related to modeling of so-called electrorheological fluids $[1,35]$ and also appear in a model for image restoration [5]. Another reason for the recent interest is that the "right" framework for variable exponent spaces was discovered: the logHölder continuity condition was found to be sufficient for many regularity properties of the spaces, starting with the local boundedness of the maximal operator [9].

Obviously, the study of trace spaces is of great importance for the theory of partial differential equations. Indeed, a partial differential equation is in many cases solvable if and only if the boundary values are in the corresponding trace space (see e.g. [16]). The first appearance of trace spaces in the context of Sobolev spaces with variable exponent $W^{1, p(\cdot)}$ is in $[14,15]$, where the solvability of the Laplace equation $-\Delta u=f$ on the half-space with given boundary values is studied. The definition of trace spaces by Diening and Růžička $[14,15]$ matches ours in Section 3. However, they avoided studying trace spaces, considering them instead as abstract objects. To describe these spaces, especially by an intrinsic norm, is the purpose of this article.

We now get back to characterizing variable exponent trace spaces. A more concrete form of the problems related to translation non-invariance can be found by looking at the well-known intrinsic characterization of the fixedexponent trace space of $W^{1, p}(\mathbb{H})$, where $\mathbb{H}$ is the open half-space $\mathbb{R}^{n} \times(0, \infty)$ : $f$ is in the trace space if and only if

$$
\int_{\mathbb{R}^{n}} \int_{\mathbb{R}^{n}} \frac{|f(x)-f(y)|^{p}}{|x-y|^{n+p-1}} d y d x<\infty .
$$

We would like to have the exponent vary with the location in the space, but clearly $p$ in the previous formula can be replaced by neither $p(x)$ nor $p(y)$. There are similar difficulties with generalizing the formulae of other fractional order spaces, such as Besov spaces or Nikol'skiü spaces. In this article we present an alternative conceptualization of the trace space problem. We try to present our approach in as simple a form as possible, in order to convey the main ideas, and hopefully to allow others to adapt them to different settings. 
The main results of this paper are summarized in the following theo$\operatorname{rem}\left({ }^{1}\right)$.

TheOREM 1.1. Let $p: \mathbb{H} \rightarrow[1, \infty)$ be a variable exponent with $1<$ $\inf p \leq \sup p<\infty$ which is globally log-Hölder continuous, i.e. assume that there exist $c>0$ and $p_{\infty}>1$ such that

$$
|p(x)-p(y)| \leq \frac{c}{\log (e+1 /|x-y|)} \quad \text { and } \quad\left|p(x)-p_{\infty}\right| \leq \frac{c}{\log (e+|x|)}
$$

for all points $x, y \in \mathbb{H}$. Then the function $f$ belongs to the trace space $\operatorname{Tr} W^{1, p(\cdot)}(\mathbb{H})$ if and only if

$$
\int_{\mathbb{R}^{n}}|f(x)|^{p(x)} d x+\int_{0}^{1} \int_{\mathbb{R}^{n}}\left(\frac{1}{r} M_{B^{n}(x, r)}^{\sharp} f\right)^{p(x)} d x d r<\infty,
$$

where $M_{B^{n}(x, r)}^{\sharp}$ denotes the sharp operator,

$$
M_{B^{n}(x, r)}^{\sharp} f=f_{B^{n}(x, r)}\left|f(y)-f_{B^{n}(x, r)} f(z) d z\right| d y .
$$

It follows that the trace space depends only on the value of the exponent on the boundary.

We prove this result in a piece-meal fashion. We start in Section 2 by introducing some standard notation and defining the variable exponent spaces. In Section 3 we define the trace space and show that it only depends on the value of the exponent on the boundary, provided the exponent is log-Hölder continuous. In Section 4 we derive the formula for the intrinsic norm of the trace space relying on a well-chosen extension of the exponent.

Many open questions still remain regarding trace theory in variable exponent spaces. We consider only extensions from $\mathbb{R}^{n}$ to the closed half-space $\overline{\mathbb{H}}$. In the fixed exponent case traces have been studied in many other settings than the half-space (see e.g. [20,21, 27]). Also, we consider only the critical smoothness, $1-1 / p(x)$. In classical notation, the spaces we consider are denoted by $W^{1-1 / p(\cdot), p(\cdot)}\left(\mathbb{R}^{n}\right)$. A future endeavor, then, is to consider also other spaces with variable smoothness on more general domains, i.e. spaces of the type $W^{s(\cdot), p(\cdot)}(\Omega)\left(^{2}\right)$.

2. Preliminaries. We will be considering the space $\mathbb{H}=\mathbb{R}^{n} \times(0, \infty)$, its closure $\overline{\mathbb{H}}$, and $\mathbb{R}^{n}$, which we view as the subspace $\mathbb{R}^{n} \times\{0\}$ of $\overline{\mathbb{H}}$. An

$\left({ }^{1}\right)$ After the completion and circulation of this paper we found out that trace spaces have been characterized by means of oscillations (see [38]). This characterization is reminiscent of ours, but has also not been previously considered in this context.

$\left({ }^{2}\right)$ Added in proof: Embeddings of variable exponent trace spaces have recently been investigated by X.-L. Fan; see [17] for details. 
analogous convention holds for arguments of functions, e.g. for $x \in \mathbb{R}^{n}$ we will sometimes write $p(x)$ instead of $p(x, 0)$. For $x \in \mathbb{R}^{n}$ and $r>0$ we denote by $B^{n}(x, r)$ the open ball in $\mathbb{R}^{n}$ with center $x$ and radius $r$. By $B^{n}$ we denote the unit ball $B^{n}(0,1)$. We use $c$ as a generic constant, i.e. a constant whose values may change from appearance to appearance. By $\chi_{A}$ we denote the characteristic function of the set $A$. We use the convention that $\chi_{A} F=0$ at all points outside $A$, regardless of whether $F$ is defined there or not.

We denote the mean value of the integrable function $f$, defined on a set $A$ of finite non-zero measure, by

$$
\langle f\rangle_{A}=f_{A} f(x) d x=\frac{1}{|A|} \int_{A} f(x) d x .
$$

For convenience we use a short-hand notation for the average over a ball:

$$
\langle f\rangle_{x, r}^{n}=\langle f\rangle_{B^{n}(x, r)} \text {. }
$$

The Hardy-Littlewood maximal operator $M$ is defined on $L_{\text {loc }}^{1}\left(\mathbb{R}^{n}\right)$ by

$$
M f(x)=\sup _{r>0}\langle|u|\rangle_{x, r}^{n} .
$$

When there is a possibility of misunderstanding, we will indicate the dimension of the underlying balls, writing $M_{(n)}$ or $M_{(n+1)}$.

Let $\Omega \subset \mathbb{R}^{n}$ be an open set. Let $p: \Omega \rightarrow[1, \infty)$ be a measurable bounded function, called a variable exponent on $\Omega$, and write $p^{+}=\operatorname{ess} \sup p(x)$ and $p^{-}=\operatorname{ess} \inf p(x)$. We define the variable exponent Lebesgue space $L^{p(\cdot)}(\Omega)$ to consist of all measurable functions $f: \Omega \rightarrow \mathbb{R}$ for which the modular

$$
\varrho_{L^{p(\cdot)}(\Omega)}(f)=\int_{\Omega}|f(x)|^{p(x)} d x
$$

is finite. We define the Luxemburg norm on this space by

$$
\|f\|_{L^{p(\cdot)}(\Omega)}=\inf \left\{\lambda>0: \varrho_{L^{p(\cdot)}(\Omega)}(f / \lambda) \leq 1\right\},
$$

which is the Minkowski functional of the absolutely convex set

$$
\left\{f \in L^{p(\cdot)}(\Omega): \varrho_{L^{p(\cdot)}(\Omega)}(f) \leq 1\right\} .
$$

In the case when $\Omega=\mathbb{R}^{n}$ we replace the $L^{p(\cdot)}\left(\mathbb{R}^{n}\right)$ in subscripts simply by $p(\cdot)$, i.e. $\|f\|_{p(\cdot)}$ stands for $\|f\|_{L^{p(\cdot)\left(\mathbb{R}^{n}\right)}}$, etc. The variable exponent Sobolev space $W^{1, p(\cdot)}(\Omega)$ is the subspace of $L^{p(\cdot)}(\Omega)$ of functions $f$ whose distributional gradient exists and satisfies $|\nabla f| \in L^{p(\cdot)}(\Omega)$. The norm

$$
\|f\|_{W^{1, p(\cdot)}(\Omega)}=\|f\|_{L^{p(\cdot)}(\Omega)}+\|\nabla f\|_{L^{p(\cdot)}(\Omega)}
$$

makes $W^{1, p(\cdot)}(\Omega)$ a Banach space.

For fixed exponent spaces we of course have a very simple relationship between norm and modular. In the variable exponent case this is not so. However, we nevertheless have the following useful property: $\varrho_{p(\cdot)}(f) \leq 1$ if 
and only if $\|f\|_{p(\cdot)} \leq 1$. This and many other basic results were proven in $[19,28]$.

We say that the exponent $p$ is (locally) log-Hölder continuous if there exists a constant $C_{\log }>0$ so that

$$
|p(x)-p(y)| \leq \frac{C_{\log }}{\log (e+1 /|x-y|)}
$$

for all points $x, y$. Some other names that have been used for these functions are 0-Hölder continuous, Dini-Lipschitz continuous and weak Lipschitz continuous. We say that the exponent $p$ is globally log-Hölder continuous if it is locally log-Hölder continuous and there exist constants $C_{\log }>0$ and $p_{\infty} \in[1, \infty)$ such that for all points $x$ we have

$$
\left|p(x)-p_{\infty}\right| \leq \frac{C_{\log }}{\log (e+|x|)} .
$$

Let us denote by $\mathcal{P}(\Omega)$ the class of globally log-Hölder continuous variable exponents on $\Omega \subset \mathbb{R}^{n}$ with $1<p^{-} \leq p^{+}<\infty$. By [8, Theorem 1.5] we know that

$$
M: L^{p(\cdot)}\left(\mathbb{R}^{n}\right) \rightarrow L^{p(\cdot)}\left(\mathbb{R}^{n}\right)
$$

is bounded if $p \in \mathcal{P}\left(\mathbb{R}^{n}\right)$. Global log-Hölder continuity is the best possible modulus of continuity to imply the boundedness of the maximal operator (see $[8,34])$. For other, weaker results see $[11,30,33]$. If the maximal operator is bounded, then it follows easily that $C_{0}^{\infty}\left(\mathbb{R}^{n}\right)$ is dense in $W^{1, p(\cdot)}\left(\mathbb{R}^{n}\right)$. In general, however, the latter condition is much weaker (see $[18,25,40])$.

3. The definition of trace spaces. Recall the definition of the trace of a $W^{1,1}$ function: if $F \in W^{1,1}(\mathbb{H}) \cap C(\overline{\mathbb{H}})$, then $\operatorname{Tr} F:=\left.F\right|_{\mathbb{R}^{n}}$ and it follows that $\|\operatorname{Tr} F\|_{L^{1}\left(\mathbb{R}^{n}\right)} \leq c\|F\|_{W^{1,1}(\mathbb{H})}$. Having defined a bounded linear operator Tr on a dense subset of $W^{1,1}$ we extend it to all of $W^{1,1}$ continuously.

Consider next a function $F \in W^{1, p(\cdot)}(\mathbb{H})$. Then $F \in W_{\text {loc }}^{1,1}(\mathbb{H})$. Thus by the previous paragraph $\operatorname{Tr} F$ is defined as a function in $L_{\text {loc }}^{1}\left(\mathbb{R}^{n}\right)$. Note that if $F \in W^{1, p(\cdot)}(\mathbb{H}) \cap C(\overline{\mathbb{H}})$, then we still have $\operatorname{Tr} F=\left.F\right|_{\mathbb{R}^{n}}$. The trace space $\operatorname{Tr} W^{1, p(\cdot)}(\mathbb{H})$ consists of the traces of all functions $F \in W^{1, p(\cdot)}(\mathbb{H})$. Notice that the elements of $\operatorname{Tr} W^{1, p(\cdot)}(\mathbb{H})$ are functions defined on $\mathbb{R}^{n}$ - to emphasize this we will always use lowercase letters for functions on $\mathbb{R}^{n}$, whereas uppercase letters will be used for functions in $\mathbb{H}$ and $\mathbb{R}^{n+1}$. The quotient norm

$$
\|f\|_{\operatorname{Tr} W^{1, p(\cdot)}(\mathbb{H})}=\inf \left\{\|F\|_{W^{1, p(\cdot)}(\mathbb{H})}: F \in W^{1, p(\cdot)}(\mathbb{H}) \text { and } \operatorname{Tr} F=f\right\}
$$

makes $\operatorname{Tr} W^{1, p(\cdot)}(\mathbb{H})$ a Banach space. The main purpose of this paper is to provide an intrinsic norm for the trace space, i.e. a norm which is defined only in terms of $f$ and not in terms of its extension $F$. 
Intuitively we would expect that this intrinsic norm only depends on $\left.p\right|_{\mathbb{R}^{n}}$ and not on $p$ on the whole space $\overline{\mathbb{H}}$. Nevertheless, the definition of $\operatorname{Tr} W^{1, p(\cdot)}(\mathbb{H})$ above is dependent on the values of $p$ on all of $\mathbb{H}$. It has often been the case that log-Hölder continuity of the exponent $p$ is a sufficient condition for variable exponent spaces to behave in a very nice way. This also turns out to hold for trace spaces:

TheOREM 3.1. Let $p_{1}, p_{2} \in \mathcal{P}(\overline{\mathbb{H}})$ with $\left.p_{1}\right|_{\mathbb{R}^{n}}=\left.p_{2}\right|_{\mathbb{R}^{n}}$. Then $\operatorname{Tr} W^{1, p_{1}(\cdot)}(\mathbb{H})$ $=\operatorname{Tr} W^{1, p_{2}(\cdot)}(\mathbb{H})$ with equivalent norms.

We will give the proof of this theorem using an extension from $W^{1, p(\cdot)}(\mathbb{H})$ to $W^{1, p(\cdot)}\left(\mathbb{R}^{n+1}\right)$. In the following proofs we also need the lower half-space $-\mathbb{H}=\mathbb{R}^{n} \times(-\infty, 0)$, its closure $-\overline{\mathbb{H}}$, and $\mathbb{R}_{\neq 0}^{n+1}=\mathbb{R}^{n} \times(\mathbb{R} \backslash\{0\})$.

Definition 3.2. Let us call $\phi$ a standard mollifier (on $\mathbb{R}^{n}$ ) if $\phi \in$ $C^{\infty}\left(\mathbb{R}^{n}\right)$ with $\phi \geq 0, \int \phi d \xi=1$ and $\operatorname{supp} \phi \subset B^{n}(0,1)$. We call $\left\{\phi_{t}\right\}$ a standard mollifier family (on $\mathbb{R}^{n}$ ) if $\phi$ is a standard mollifier and $\phi_{t}(\xi)=$ $t^{-n} \phi(\xi / t)$.

Note that if $p \in \mathcal{P}\left(\mathbb{R}^{n}\right)$ and $\left\{\phi_{t}\right\}$ is a standard mollifier family, then $\phi_{t} * f \rightarrow f$ in $W^{1, p(\cdot)}(\Omega)$ for all $f \in W^{1, p(\cdot)}(\Omega)$ (see $[6,9,36,37]$ ).

TheOREM 3.3. Let $p \in \mathcal{P}\left(\mathbb{R}^{n+1}\right)$. Then there exists a bounded, linear extension operator $\mathcal{E}: W^{1, p(\cdot)}(\mathbb{H}) \rightarrow W^{1, p(\cdot)}\left(\mathbb{R}^{n+1}\right)$.

Proof. Let $F \in W^{1, p(\cdot)}(\mathbb{H})$. It follows from $p \in \mathcal{P}\left(\mathbb{R}^{n+1}\right)$ that $C_{0}^{\infty}(\overline{\mathbb{H}})$ is dense in $W^{1, p(\cdot)}(\mathbb{H})$, hence it suffices to prove the claim for $F \in C^{\infty}(\mathbb{H})$. Let $\left\{\phi_{t}\right\}$ be a standard mollifier family on $\mathbb{R}^{n+1}$. Then we define $\mathcal{E} F: \mathbb{R}^{n+1} \rightarrow \mathbb{R}$ by

$$
\mathcal{E} F(x, t):= \begin{cases}F(x, t) & \text { for } t \geq 0 \\ \left(\phi_{|t|} * F\right)(x,|t|) & \text { for } t<0 .\end{cases}
$$

We have to show that $\mathcal{E} F \in W^{1, p(\cdot)}\left(\mathbb{R}^{n+1}\right)$ with bounded norm. Obviously, $\mathcal{E} F \in C^{\infty}(-\mathbb{H})$. In the following we write $\xi:=(x, t) \in-\mathbb{H}$ and $\xi^{\prime}:=(x,|t|)$. We directly estimate

$$
\begin{aligned}
|(\mathcal{E} F)(\xi)| & =\left|\left(\phi_{|t|} * F\right)\left(\xi^{\prime}\right)\right| \leq c(\phi) M_{(n+1)}\left(\chi_{\mathbb{H}} F\right)\left(\xi^{\prime}\right), \\
\left|\nabla_{x}(\mathcal{E} F)(\xi)\right| & =\left|\left(\phi_{|t|} * \nabla_{x} F\right)\left(\xi^{\prime}\right)\right| \leq c(\phi) M_{(n+1)}\left(\chi_{\mathbb{H}} \nabla_{x} F\right)\left(\xi^{\prime}\right) .
\end{aligned}
$$

For the $t$-derivative we need a slightly more involved calculation: for all $\xi \equiv(x, t) \in-\mathbb{H}$ and $a \in \mathbb{R}$ we have

$$
\begin{aligned}
\partial_{t}(\mathcal{E} F)(\xi) & =\frac{\partial}{\partial t}\left(\phi_{|t|} * F\right)\left(\xi^{\prime}\right)=\frac{\partial}{\partial t}\left(\phi_{|t|} *(F-a)\right)\left(\xi^{\prime}\right) \\
\quad= & \int_{\mathbb{R}^{n+1}}\left[\frac{n+1}{|t|} \phi_{|t|}\left(\xi^{\prime}-\eta\right)+\frac{1}{|t|^{2}}(\nabla \phi)_{|t|}\left(\xi^{\prime}-\eta\right) \cdot\left(\xi^{\prime}-\eta\right)\right](F(\eta)-a) d \eta
\end{aligned}
$$


where $(\nabla \phi)_{r}(\eta):=r^{-n-1} \nabla \phi(\eta / r)$ for $r>0$. Setting $a=\langle F\rangle_{\xi^{\prime},|t|}^{n+1}$ we find that

$$
\begin{aligned}
& \left|\partial_{t}(\mathcal{E} F)(\xi)\right| \\
& \quad \leq \int_{B^{n+1}\left(\xi^{\prime},|t|\right)}\left(\frac{n+1}{|t|^{n+2}}\|\phi\|_{\infty}+\frac{1}{|t|^{n+3}}\|\nabla \phi\|_{\infty}\left|\xi^{\prime}-\eta\right|\right)\left|F(\eta)-\langle F\rangle_{\xi^{\prime},|t|}^{n+1}\right| d \eta \\
& \quad \leq|t|^{-n-2}\left((n+1)\|\phi\|_{\infty}+\|\nabla \phi\|_{\infty}\right) \int_{B^{n+1}\left(\xi^{\prime},|t|\right)}\left|F(\eta)-\langle F\rangle_{\xi^{\prime},|t|}^{n+1}\right| d \eta \\
& \quad=\frac{c(\phi)}{t} \int_{B^{n+1}\left(\xi^{\prime},|t|\right)}\left|F(\eta)-\langle F\rangle_{\xi^{\prime},|t|}^{n+1}\right| d \eta .
\end{aligned}
$$

Then the Poincaré inequality implies that

$$
\left|\partial_{t}(\mathcal{E} F)(\xi)\right| \leq c(\phi)\langle|\nabla F|\rangle_{\xi^{\prime},|t|}^{n+1} \leq c(\phi) M_{(n+1)}\left(\chi_{\mathbb{H}} \nabla F\right)\left(\xi^{\prime}\right) .
$$

Overall, we have shown that

$$
|(\mathcal{E} F)(\xi)| \leq c M_{(n+1)}\left(\chi_{\mathbb{H}} F\right)\left(\xi^{\prime}\right), \quad|\nabla(\mathcal{E} F)(\xi)| \leq c M_{(n+1)}\left(\chi_{\mathbb{H}} \nabla F\right)\left(\xi^{\prime}\right)
$$

for all $\xi \equiv(x, t) \in-\mathbb{H}$. Since $M_{(n+1)}$ is continuous from $L^{p(\cdot)}\left(\mathbb{R}^{n+1}\right)$ to $L^{p(\cdot)}\left(\mathbb{R}^{n+1}\right)$, this pointwise inequality implies that

$$
\begin{aligned}
\|\mathcal{E} F\|_{W^{1, p(\cdot)}(-\mathbb{H})} & \leq c\left\|M_{(n+1)} F\right\|_{L^{p(\cdot)}(\mathbb{H})}+c\left\|M_{(n+1)}\left(\chi_{\mathbb{H}} \nabla F\right)\right\|_{L^{p(\cdot)}(\mathbb{H})} \\
& \leq c\|F\|_{W^{1, p(\cdot)}(\mathbb{H})} .
\end{aligned}
$$

It remains to show that $\mathcal{E} F$ has a distributional gradient in $\mathbb{R}^{n+1}$, which, by [41, Theorem 2.1.4], follows once we show that $\mathcal{E} F$ is absolutely continuous on lines (ACL). Recall that this means (by definition) that the set of values $x \in \mathbb{R}^{n}$ for which the function $t \mapsto \mathcal{E} F(x, t)$ is not absolutely continuous on $\mathbb{R}$ has $n$-measure zero, and similarly for all the other co-ordinate directions. We easily see that $\mathcal{E} F \in C\left(\mathbb{R}^{n+1}\right)$, and that $\mathcal{E} F$ is ACL on both $\mathbb{H}$ and $-\mathbb{H}$, from which it directly follows that $\mathcal{E} F \in \operatorname{ACL}\left(\mathbb{R}^{n+1}\right)$, so $\nabla \mathcal{E} F$ exists in the distributional sense in the whole space.

We now show how Theorem 3.3 implies Theorem 3.1.

Proof of Theorem 3.1. Define $q(x, t):=p_{1}(x, t)$ for $t \geq 0$ and $q(x, t):=$ $p_{2}(x,-t)$ for $t<0$. Then $q \in \mathcal{P}\left(\mathbb{R}^{n+1}\right)$. By Theorem 3.3 there exist bounded, linear extensions

$$
\begin{aligned}
& \mathcal{E}_{1}: W^{1, q(\cdot)}(\mathbb{H}) \rightarrow W^{1, q(\cdot)}\left(\mathbb{R}^{n+1}\right), \\
& \mathcal{E}_{2}: W^{1, q(\cdot)}(-\mathbb{H}) \rightarrow W^{1, q(\cdot)}\left(\mathbb{R}^{n+1}\right) .
\end{aligned}
$$

This directly implies $\operatorname{Tr} W^{1, q(\cdot)}(-\mathbb{H})=\operatorname{Tr} W^{1, q(\cdot)}(\mathbb{H})$ with equivalence of norms. The identities $\operatorname{Tr} W^{1, q(\cdot)}(\mathbb{H})=\operatorname{Tr} W^{1, p(\cdot)}(\mathbb{H})$ and $\operatorname{Tr} W^{1, q(\cdot)}(-\mathbb{H})=$ $\operatorname{Tr} W^{1, p(\cdot)}(\mathbb{H})$ (by reflection) conclude the proof of the theorem. 
REMARK 3.4. The first author has previously proven an extension theorem for variable exponent spaces (see [10, Theorem 4.2]). The difference between that result and Theorem 3.3 is the following: in Theorem 3.3 the exponent $p$ is already given outside of $\overline{\mathbb{H}}$ while in [10] the exponent $p$ had to be extended from $\overline{\mathbb{H}}$ to $\mathbb{R}^{n+1}$ in a special way.

Recall the definition of the Sobolev space of functions with zero boundary value: the space $W_{0}^{1, p(\cdot)}(\mathbb{H})$ is the completion of $C_{0}^{\infty}(\mathbb{H})$ in $W^{1, p(\cdot)}(\mathbb{H})$. (Other definitions are better when smooth functions are not dense; see [21-23].) We next characterize $W_{0}^{1, p(\cdot)}(\mathbb{H})$ in terms of traces.

TheOREM 3.5. Suppose that $p \in \mathcal{P}(\overline{\mathbb{H}})$ and let $F \in W^{1, p(\cdot)}(\mathbb{H})$. Then $F \in W_{0}^{1, p(\cdot)}(\mathbb{H})$ if and only if $\operatorname{Tr} F=0$.

Proof. Suppose first that $F \in W^{1, p(\cdot)}(\mathbb{H})$ with $\operatorname{Tr} F=0$. We extend $p$ to $-\mathbb{H}$ by reflection. Since $W^{1, p(\cdot)}(\mathbb{H}) \hookrightarrow W_{\text {loc }}^{1,1}(\mathbb{H})$, it follows by classical theory that $F$ extended by 0 to the lower half-space $-\mathbb{H}$ is differentiable in the sense of distributions in $\mathbb{R}^{n+1}$, and hence $F$ is in $W^{1, p(\cdot)}\left(\mathbb{R}^{n+1}\right)$. Now let $\phi$ be a standard mollifier with support in $B^{n+1}\left(e_{n+1} / 2,1 / 3\right)$, where $e_{n+1}$ denotes the $(n+1)$ st unit vector. Then $\phi_{r} *\left(F \chi_{B^{n+1}(0,1 / r)}\right)$ has compact support in $\mathbb{H}$ and is smooth. Since $p \in \mathcal{P}\left(\mathbb{R}^{n+1}\right)$, it follows that $\phi_{r} *\left(F \chi_{B^{n+1}(0,1 / r)}\right) \rightarrow F$ in $W^{1, p(\cdot)}\left(\mathbb{R}^{n+1}\right)$ as $r \rightarrow 0$, so $F \in W_{0}^{1, p(\cdot)}(\mathbb{H})$.

For the converse, if $F \in W_{0}^{1, p(\cdot)}(\mathbb{H})$, then, by definition, $F=\lim \phi_{i}$ in $W^{1, p(\cdot)}(\mathbb{H})$, where $\phi_{i} \in C_{0}^{\infty}(\mathbb{H})$. Since $\operatorname{Tr} \phi_{i}=\left.\phi_{i}\right|_{\mathbb{R}^{n}} \equiv 0$, the claim follows by continuity of $\operatorname{Tr}: W^{1, p(\cdot)}(\mathbb{H}) \rightarrow \operatorname{Tr} W^{1, p(\cdot)}(\mathbb{H})$.

REMARK 3.6. The previous theorem also holds under the weaker assumption that the exponent is such that smooth functions are dense in the Sobolev space. The proof is, however, more complicated, and we refer the reader to [12] for details.

The following simple result was proven recently in [7, Lemma 4.3]. We include the proof for completeness, since our proof is much shorter than that in [7].

Proposition 3.7. Let $X \subset \mathbb{R}^{n}$. If $p \in \mathcal{P}(X)$, then there exists an extension $\widetilde{p} \in \mathcal{P}\left(\mathbb{R}^{n}\right)$ with the same log-Hölder constant and the same upper and lower bounds.

Proof. Let $C_{\log }>0$ and $p_{\infty} \geq 1$ be such that

$$
|p(x)-p(y)| \leq \frac{C_{\log }}{\log (e+1 /|x-y|)} \quad \text { and } \quad\left|p(x)-p_{\infty}\right| \leq \frac{C_{\log }}{\log (e+|x|)}
$$

for all points $x, y \in X$. 
Since $t \mapsto 1 / \log (e+1 / t)$ is concave on $(0, \infty)$, we can use a McShane-type maximal extension [31] of $p$. More precisely, we define

$$
\bar{p}(y)=\sup _{x \in X}\left(p(x)-\frac{C_{\log }}{\log (e+1 /|x-y|)}\right)
$$

for $y \in \mathbb{R}^{n}$. Then we truncate $\bar{p}(x)$ from above and below by

$$
\min \left\{p_{X}^{+}, p_{\infty}+\frac{C_{\log }}{\log (e+|x|)}\right\} \text { and } \max \left\{p_{X}^{-}, p_{\infty}-\frac{C_{\log }}{\log (e+|x|)}\right\},
$$

respectively. Since the constant function and $x \mapsto(\log (e+|x|))^{-1}$ both have log-Hölder constant less than one, we see that the truncation does not affect the local log-Hölder constant of $\bar{p}$. Therefore the truncated $\bar{p}$ is the exponent $\widetilde{p}$ we are looking for.

The above proposition and Theorem 3.1 imply that the following definition is sensible (up to equivalence of norms).

Definition 3.8. Let $p \in \mathcal{P}\left(\mathbb{R}^{n}\right)$ and let $q \in \mathcal{P}(\mathbb{H})$ be an arbitrary extension of $p$. Then we define an intrinsic trace space by

$$
\left(\operatorname{Tr} W^{1, p(\cdot)}\right)\left(\mathbb{R}^{n}\right):=\operatorname{Tr} W^{1, q(\cdot)}(\mathbb{H}) .
$$

REMARK 3.9. When $p \in \mathcal{P}\left(\mathbb{R}^{n}\right)$, Theorem 3.1 simplifies studying the space $\left(\operatorname{Tr} W^{1, p(\cdot)}\right)\left(\mathbb{R}^{n}\right)$ significantly. Indeed, for $x \in \mathbb{R}^{n}$ and $t \in[0,2]$ define $q(x, t):=p(x)$. Then $q$ is globally log-Hölder continuous on $\mathbb{R}^{n} \times[0,2]$ with $1<q^{-} \leq q^{+}<\infty$. As in Lemma 3.7 we can extend $q$ to the set $\overline{\mathbb{H}}$ so that $q \in \mathcal{P}(\overline{\mathbb{H}})$. We have $\left(\operatorname{Tr} W^{1, p(\cdot)}\right)\left(\mathbb{R}^{n}\right)=\operatorname{Tr} W^{1, q(\cdot)}(\mathbb{H})$. So we can always assume that the exponent $q(x, t)$ is independent of $t$ when $t \in[0,2]$.

4. Intrinsic characterization of the trace space. For a function $f \in L_{\text {loc }}^{1}\left(\mathbb{R}^{n}\right)$ we define the sharp operator by

$$
M_{B^{n}(x, r)}^{\sharp} f=f_{B^{n}(x, r)}\left|f(y)-\langle f\rangle_{x, r}^{n}\right| d y .
$$

Using the triangle inequality it is easy to show that

$$
M_{B^{n}(x, r)}^{\sharp} f \leq f_{B^{n}(x, r)} f_{B^{n}(x, r)}|f(y)-f(z)| d y d z \leq 2 M_{B^{n}(x, r)}^{\sharp} f .
$$

We define the trace modular $\varrho_{\operatorname{Tr}, p(\cdot)}$ by

$$
\varrho_{\operatorname{Tr}, p(\cdot)}(f)=\int_{\mathbb{R}^{n}}|f(x)|^{p(x)} d x+\int_{0 \mathbb{R}^{n}}^{1}\left(\frac{1}{r} M_{B^{n}(x, r)}^{\sharp} f\right)^{p(x)} d x d r .
$$

Obviously, $\varrho_{\operatorname{Tr}, p(\cdot)}$ is convex. Thus

$$
\|f\|_{\operatorname{Tr}, p(\cdot)}:=\inf \left\{\lambda>0: \varrho_{\operatorname{Tr}, p(\cdot)}(f / \lambda) \leq 1\right\}
$$


is a norm, since it is the Minkowski functional of the absolutely convex set $\left\{f: \varrho_{\operatorname{Tr}, p(\cdot)}(f) \leq 1\right\}$.

The following theorem characterizes the traces of $W^{1, p(\cdot)}(\mathbb{H})$-functions and completes the proof of Theorem 1.1.

TheOREM 4.2. Let $p \in \mathcal{P}(\overline{\mathbb{H}})$ and let $f \in L_{\text {loc }}^{1}\left(\mathbb{R}^{n}\right)$. Then $f$ belongs to $\operatorname{Tr} W^{1, p(\cdot)}(\mathbb{H})$ if and only if $\|f\|_{\operatorname{Tr}, p(\cdot)}<\infty$, or, equivalently,

$$
\int_{\mathbb{R}^{n}}|f(x)|^{p(x)} d x+\int_{0}^{1} \int_{\mathbb{R}^{n}}\left(\frac{1}{r} M_{B^{n}(x, r)}^{\sharp} f\right)^{p(x)} d x d r<\infty,
$$

where $p(x):=p(x, 0)$. Moreover, $\|f\|_{\operatorname{Tr}, p(\cdot)}$ is equivalent to the quotient norm $\|f\|_{\operatorname{Tr} W^{1, p(\cdot)(\mathbb{H})}} \cdot$

To prove the theorem we have to show two things. First, for $F \in W^{1, p(\cdot)}(\mathbb{H})$

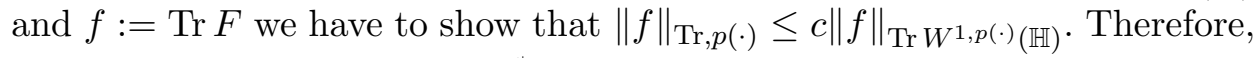
we have to estimate $|f|$ and $M_{B^{n}(x, t)}^{\sharp} f$ in terms of $|F|$ and $|\nabla F|$. Second, for $f \in \operatorname{Tr} W^{1, p(\cdot)}(\mathbb{H})$ we have to show the existence of some $F \in W^{1, p(\cdot)}(\mathbb{H})$ with $\operatorname{Tr} F=f$ and $\|F\|_{W^{1, p(\cdot)(\mathbb{H})}} \leq c\|f\|_{\operatorname{Tr}, p(\cdot)}$. We will define the extension $F$ by $F(x, t):=\left(\phi_{t} * f\right)(x)$ for $x \in \mathbb{R}^{n}$ and $t>0$, where $\left(\phi_{t}\right)$ is a standard mollifier family in $\mathbb{R}^{n}$. In order to estimate $\|F\|_{W^{1, p(\cdot)(\mathbb{H})}}$ we need to estimate $|F|$ and $|\nabla F|$ in terms of $|f|$ and $M_{B^{n}(x, t)}^{\sharp} f$. The following two lemmas provide these estimates.

LEMma 4.3. There exists a constant $c_{1}>0$ so that

$$
M_{B^{n}(z, r)}^{\sharp} \operatorname{Tr} F \leq c_{1} r \quad f_{B^{n+1}((z, 0), r)} \chi_{\mathbb{H}}(\xi)|\nabla F(\xi)| d \xi
$$

for all $z \in \mathbb{R}^{n}, r>0$ and $F \in W^{1,1}\left(B^{n+1}((z, 0), r)\right)$.

Proof. Since smooth functions are dense in $W^{1,1}\left(B^{n+1}(z, r)\right)$ it suffices to prove the claim for smooth $F$. As usual we write $f=\operatorname{Tr} F=\left.F\right|_{\mathbb{R}^{n}}$. Let us estimate $|f(x)-f(y)|$ for $x, y \in \mathbb{R}^{n}$ by integrating the gradient over the path $\gamma_{\zeta}=[x, \zeta] \cup[\zeta, y]$ for $\zeta \in \mathbb{H}$ :

$$
|f(x)-f(y)| \leq \int_{\gamma_{\zeta}}|\nabla F(\xi)| d \xi .
$$

Define $B_{x, y}=B^{n+1}\left(\frac{x+y}{2}+\frac{|x-y|}{4} e_{n+1}, \frac{|x-y|}{8}\right) \cap P$, where $P$ is the mid-point normal plane of the segment $[x, y]$ and let $A_{x, y}=\bigcup_{\zeta \in B_{x, y}} \gamma_{\zeta}$. Next we take the average integral of (4.4) over $\zeta \in B_{x, y}$. This so-called Riesz potential estimate (e.g. [26]) yields

$$
|f(x)-f(y)| \leq c \int_{A_{x, y}}|\nabla F(\xi)|\left(|x-\xi|^{-n}+|y-\xi|^{-n}\right) d \xi .
$$


Let $z \in \mathbb{R}^{n}$ and $r>0$. Using the previous estimate together with (4.1) gives

$$
M_{B^{n}(z, r)}^{\sharp} f \leq c \underset{B^{n}(z, r)}{f} \underset{B^{n}(z, r)}{f} \int_{A_{x, y}}|\nabla F(\xi)| t^{-n} d \xi d x d y,
$$

where $t$ denotes the $(n+1)$ st co-ordinate of $\xi$ and we used the fact that $t \leq \min \{|y-\xi|,|x-\xi|\}$ when $\xi \in A_{x, y}$.

The set $A_{x, y}$ consists of two cones, one emanating from $y$ and the other from $x$, denoted by $A_{x, y}^{\prime}$ and $A_{x, y}^{\prime \prime}$, respectively. By symmetry, we see that we can replace $A_{x, y}$ by $A_{x, y}^{\prime}$ in (4.5). We want to swap the order of integrations. So suppose that $\xi \in A_{x, y}^{\prime}$. Then certainly $\xi \in B^{n+1}(z, r)$. Also, $\xi$ lies in a cone emanating from $y$ whose direction depends on $x-y$. Thus we see that $y$ lies in the cone emanating from $\xi$ with the same base-angle but opposite direction. This means that for a fixed $\xi$ the variable $y$ varies in a ball $B^{n}\left(w, c^{\prime} t\right)$ where $\xi=(w, t)$ and $c^{\prime}>0$ depends only on the dimension $n$. Hence

$$
\begin{aligned}
M_{B^{n}(z, r)}^{\sharp} f & \leq c r^{-2 n} \int_{B^{n}(z, r)} \int_{B^{n}(z, r)} \int_{A_{x, y}^{\prime}}|\nabla F(\xi)| t^{-n} d \xi d x d y \\
& \leq c r^{-2 n} \int_{B^{n+1}((z, 0), r)} \chi_{\mathbb{H}}(\xi)|\nabla F(\xi)| t^{-n} \int_{B^{n}\left(w, c^{\prime} t\right)} \int_{B^{n}(z, r)} d x d y d \xi \\
& =c r \int_{B^{n+1}((z, 0), r)} \chi_{\mathbb{H}}(\xi)|\nabla F(\xi)| d \xi .
\end{aligned}
$$

This proves the lemma.

The proof of the next lemma is essentially the same as the proof of Theorem 3.3, so it is omitted here.

Lemma 4.6. Let $\left\{\phi_{t}\right\}$ be a standard mollifier family on $\mathbb{R}^{n}$. Let $f \in$ $L_{\text {loc }}^{1}\left(\mathbb{R}^{n}\right)$ and define $F(x, t):=\phi_{t} * f(x)$ for $x \in \mathbb{R}^{n}$ and $t \in(0, \infty)$. Then there exists a constant $c_{2}$ depending only on $\phi$ and $n$ such that, for all $x \in \mathbb{R}^{n}$ and $t \in(0, \infty)$,

$$
|F(x, t)| \leq c_{2}\langle|f|\rangle_{x, t}^{n}, \quad|\nabla F(x, t)| \leq \frac{c_{2}}{t} M_{B^{n}(x, t)}^{\sharp} f .
$$

Thus we are ready for the proof of the main result.

Proof of Theorem 4.2. Due to Theorem 3.1 and Remark 3.9 we can assume without loss of generality that $p(x, t)=p(x, 0)=p(x)$ for $x \in \mathbb{R}^{n}$ and $t \in[0,2]$.

Let $\left\{\phi_{t}\right\}$ be a standard mollifier family on $\mathbb{R}^{n}$, and let $f \in \operatorname{Tr} W^{1, p(\cdot)}(\mathbb{H})$ with $\|f\|_{\operatorname{Tr} W^{1, p(\cdot)(\mathbb{H})}} \leq 1$, or equivalently, with

$$
\int_{\mathbb{R}^{n}}|f(x)|^{p(x)} d x+\int_{0}^{1} \int_{\mathbb{R}^{n}}\left(\frac{1}{r} M_{B^{n}(x, r)}^{\sharp} f\right)^{p(x)} d x d r \leq 1 .
$$


We have to show the existence of an extension $F \in W^{1, p(\cdot)}(\mathbb{H})$ which satisfies $\|F\|_{W^{1, p(\cdot)(\mathbb{H})}} \leq c$, where $c$ is independent of $f$. As mentioned above, we would like to consider the extension $(x, t) \mapsto \phi_{t} * f(x)$. But in order to avoid difficulties as $t \rightarrow \infty$ we cut off the part for large $t$. Let $\psi \in C_{0}^{\infty}([0, \infty))$ with $\chi_{B^{1}(0,1 / 2)} \leq \psi \leq \chi_{B^{1}(0,1)}$. Then our extension $F$ is given by $F(x, t):=$ $\phi_{t} * f(x) \psi(t)$.

We now estimate the norm of $F$ in $W^{1, p(\cdot)}(\mathbb{H})$. Using Lemma 4.6 and noting that $\langle|f|\rangle_{x, t}^{n} \leq M f(x)$, we find that

$$
\varrho_{L^{p(\cdot)}(\mathbb{H})}(F)=\int_{0}^{1} \int_{\mathbb{R}^{n}}|F(x, t)|^{p(x)} d x d t \leq c \int_{\mathbb{R}^{n}} M f(x)^{p(x)} d x .
$$

Our assumptions on $p$ imply that the maximal operator is bounded on $L^{p(\cdot)}\left(\mathbb{R}^{n}\right)$. Since $\varrho_{p(\cdot)}(f) \leq 1$, it follows from the previous inequality that $\varrho_{L^{p(\cdot)(\mathbb{H})}}(F) \leq c$. We move to the norm of the gradient. Using Lemma 4.6 again, we estimate

$$
\begin{aligned}
\varrho_{L^{p(\cdot)(\mathbb{H})}}(\nabla F) & =\int_{0}^{1} \int_{\mathbb{R}^{n}}|\nabla F(x, t)|^{p(x)} d x d t \\
& \leq c(\psi) \int_{0}^{1} \int_{\mathbb{R}^{n}}\left|\frac{1}{t} M_{B^{n}(x, t)}^{\sharp} f\right|^{p(x)} d x d t \leq c .
\end{aligned}
$$

Thus we have shown that $F \in W^{1, p(\cdot)}(\mathbb{H})$. Furthermore, it follows easily that $f=\operatorname{Tr} F$, so we have proved one of the implications in the theorem.

To prove the opposite implication, we use the density of smooth functions and restrict ourselves without loss of generality to $F \in W^{1, p(\cdot)}(\mathbb{H}) \cap C^{\infty}(\overline{\mathbb{H}})$. Replacing $F$ by $F \psi$, where $\psi$ is as above, we see that it suffices to consider $F$ supported in $\mathbb{R}^{n} \times[0,1]$. By homogeneity, it suffices to consider the case $\|F\|_{W^{1, p(\cdot)}(\mathbb{H})} \leq 1$ and to prove $\|f\|_{\operatorname{Tr}, p(\cdot)} \leq C$. Since $p$ is bounded, the latter condition is equivalent to $\varrho_{\operatorname{Tr}, p(\cdot)}(f) \leq C$, which is what we now prove. Define $f:=\operatorname{Tr} F$. We find that

$$
|f(x)|=|F(x, 0)| \leq \int_{0}^{1}|\nabla F(x, t)| d t .
$$

Hence using Jensen's inequality we get

$$
|f(x)|^{p(x)} \leq \int_{0}^{1}|\nabla F(x, t)|^{p(x)} d t,
$$

and, integrating over $x \in \mathbb{R}^{n}$, 


$$
\varrho_{p(\cdot)}(f)=\int_{\mathbb{R}^{n}}|f(x)|^{p(x)} d x \leq \int_{\mathbb{R}^{n}} \int_{0}^{1}|\nabla F(x, t)|^{p(x)} d t d x=\varrho_{L^{p(\cdot)(\mathbb{H})}}(\nabla F) .
$$

Thus we have bounded the $L^{p(\cdot)}$ part of the trace norm.

Since $f=\operatorname{Tr} F$, by Lemma 4.3 we get

$$
\begin{aligned}
& \int_{0}^{1} \int_{\mathbb{R}^{n}}\left(\frac{1}{r} M_{B^{n}(x, r)}^{\sharp} f\right)^{p(x)} d x d r \\
& \leq c \int_{0 \mathbb{R}^{n}}^{1}\left(f_{B^{n+1}((x, 0), r)} \chi_{\mathbb{H}}(\xi)|\nabla F(\xi)| d \xi\right)^{p(x)} d x d r \\
& \leq c \int_{0 \mathbb{R}^{n}}^{1}\left(f_{B^{n+1}((x, r), 2 r)} \chi_{\mathbb{H}}(\xi)|\nabla F(\xi)| d \xi\right)^{p(x)} d x d r \\
& \leq c \int_{\mathbb{R}^{n} \times[0,1]}\left(M_{(n+1)}\left(\chi_{\mathbb{H}}|\nabla F|\right)(\eta)\right)^{p(\eta)} d \eta
\end{aligned}
$$

Extending the exponent to the lower half-space by reflection, we immediately see that $p \in \mathcal{P}\left(\mathbb{R}^{n+1}\right)$ and

$$
\int_{0}^{1} \int_{\mathbb{R}^{n}}\left(\frac{1}{r} M_{B^{n}(x, r)}^{\sharp} f\right)^{p(x)} d x d r \leq c \int_{\mathbb{R}^{n+1}}\left(M_{(n+1)}\left(\chi_{\mathbb{H}}|\nabla F|\right)(\xi)\right)^{p(\xi)} d \xi .
$$

Since the maximal operator is bounded on $L^{p(\cdot)}\left(\mathbb{R}^{n+1}\right)$, the right-hand side of the previous inequality is bounded by a constant, which concludes the proof.

Acknowledgments. We would like to thank the referee for a careful reading and several useful suggestions.

\section{References}

[1] E. Acerbi and G. Mingione, Regularity results for stationary electro-rheological fluids, Arch. Ration. Mech. Anal. 164 (2002), 213-259.

$[2]-$ - - Gradient estimates for the $p(x)$-Laplacean system, J. Reine Angew. Math. 584 (2005), 117-148.

[3] R. Adams, Sobolev Spaces, Pure Appl. Math. 65, Academic Press, New York, 1975.

[4] R. Adams and J. Fournier, Sobolev Spaces, 2nd ed., Pure Appl. Math. 140, Academic Press, New York, 2003.

[5] Y. Chen, S. Levine and R. Rao, Variable exponent, linear growth functionals in image restoration, SIAM J. Appl. Math. 66 (2006), 1383-1406.

[6] D. Cruz-Uribe and A. Fiorenza, Approximate identities in variable $L^{p}$ spaces, Math. Nachr. 280 (2007), 256-270.

[7] D. Cruz-Uribe, A. Fiorenza, J. M. Martell and C. Pérez, The boundedness of classical operators on variable $L^{p}$ spaces, Ann. Acad. Sci. Fenn. Math. 31 (2006), 239-264. 
[8] D. Cruz-Uribe, A. Fiorenza and C. J. Neugebauer, The maximal function on variable $L^{p}$ spaces, Ann. Acad. Sci. Fenn. Math. 28 (2003), 223-238; corrections, 29 (2004), $247-249$.

[9] L. Diening, Maximal function on generalized Lebesgue spaces $L^{p(\cdot)}$, Math. Inequal. Appl. 7 (2004), 245-253.

[10] —, Riesz potential and Sobolev embeddings on generalized Lebesgue and Sobolev spaces $L^{p(\cdot)}$ and $W^{k, p(\cdot)}$, Math. Nachr. 268 (2004), 31-43.

[11] - Maximal function on Musielak-Orlicz spaces and generalized Lebesgue spaces, Bull. Sci. Math. 129 (2005), 657-700.

[12] L. Diening and P. Hästö, Further results on variable exponent trace spaces, in: Proceedings of the 5th ISAAC, to appear.

[13] L. Diening, P. Hästö and A. Nekvinda, Open problems in variable exponent Lebesgue and Sobolev spaces, in: Function Spaces, Differential Operators and Nonlinear Analysis (Milovy, 2004), P. Drábek and J. Rákosník (eds.), Math. Inst. Acad. Sci. Czech Republic, Praha, 2005, 38-58.

[14] L. Diening and M. Růžička, Integral operators on the halfspace in generalized Lebesgue spaces $L^{p(\cdot)}$, part I, J. Math. Anal. Appl. 298 (2004), 559-571.

[15] - - - Integral operators on the halfspace in generalized Lebesgue spaces $L^{p(\cdot)}$, part II, ibid., 572-588.

[16] L. C. Evans, Partial Differential Equations, Grad. Stud. Math. 19, Amer. Math. Soc., Providence, RI, 1998.

[17] X.-L. Fan, Boundary trace embedding theorems for variable exponent Sobolev spaces, J. Math. Anal. Appl., to appear.

[18] X. L. Fan, S. Y. Wang and D. Zhao, Density of $C^{\infty}(\Omega)$ in $W^{1, p(x)}(\Omega)$ with discontinuous exponent $p(x)$, Math. Nachr. 279 (2006), 142-149.

[19] X. L. Fan and D. Zhao, On the spaces $L^{p(x)}(\Omega)$ and $W^{m, p(x)}(\Omega)$, J. Math. Anal. Appl. 263 (2001), 424-446.

[20] E. Gagliardo, Caratterizzazioni delle tracce sulla frontiera relative ad alcune classi di funzioni in $n$ variabili, Rend. Sem. Mat. Univ. Padova 27 (1957), 284-305.

[21] P. Hajłasz and O. Martio, Traces of Sobolev functions on fractal type sets and characterization of extension domains, J. Funct. Anal. 143 (1997), 221-246.

[22] P. Harjulehto, Variable exponent Sobolev spaces with zero boundary values, Math. Bohem. 132 (2007), 125-136.

[23] P. Harjulehto and P. Hästö, Lebesgue points in variable exponent spaces, Ann. Acad. Sci. Fenn. Math. 29 (2004), 295-306.

[24] P. Harjulehto, P. Hästö, M. Koskenoja and S. Varonen, Sobolev capacity on the space $W^{1, p(\cdot)}\left(\mathbb{R}^{n}\right)$, J. Funct. Spaces Appl. 1 (2003), 17-33.

[25] P. Hästö, On the density of continuous functions in variable exponent Sobolev space, Rev. Mat. Iberoamer. 23 (2007), 213-234.

[26] J. Heinonen, Nonsmooth calculus, Bull. Amer. Math. Soc. (N.S.) 44 (2007), 163-232.

[27] A. Jonsson, Besov spaces on closed subsets of $\mathbb{R}^{n}$, Trans. Amer. Math. Soc. 341 (1994), 355-370.

[28] O. Kováčik and J. Rákosník, On spaces $L^{p(x)}$ and $W^{k, p(x)}$, Czechoslovak Math. J. 41 (116) (1991), 592-618.

[29] H.-G. Leopold, Embedding of function spaces of variable order of differentiation in function spaces of variable order of integration, ibid. 49 (124) (1999), 633-644.

[30] A. Lerner, Some remarks on the Hardy-Littlewood maximal function on variable $L^{p}$ spaces, Math. Z. 251 (2005), 509-521.

[31] E. J. McShane, Extension of range of functions, Bull. Amer. Math. Soc. 40 (1934), $837-842$. 
[32] J. Musielak, Orlicz Spaces and Modular Spaces, Lecture Notes in Math. 1034, Springer, Berlin, 1983.

[33] A. Nekvinda, Hardy-Littlewood maximal operator on $L^{p(x)}\left(\mathbb{R}^{n}\right)$, Math. Inequal. Appl. 7 (2004), 255-266.

[34] L. Pick and M. Rǔžička, An example of a space $L^{p(x)}$ on which the Hardy-Littlewood maximal operator is not bounded, Expo. Math. 19 (2001), 369-371.

[35] M. Růžička, Electrorheological Fluids: Modeling and Mathematical Theory, Springer, Berlin, 2000.

[36] S. Samko, Convolution type operators in $L^{p(x)}$, Integral Transform. Spec. Funct. 7 (1998), 123-144.

[37] - Convolution and potential type operators in $L^{p(x)}\left(\mathbb{R}^{n}\right)$, ibid., 261-284.

[38] H. Triebel, Theory of Function Spaces II, Monogr. Math. 84, Birkhäuser, Basel, 1992.

[39] V. V. Zhikov, Averaging of functionals of the calculus of variations and elasticity theory, Izv. Akad. Nauk SSSR Ser. Mat. 50 (1986), 675-710 (in Russian); English transl.: Math. USSR-Izv. 29 (1987), 33-66.

[40] - On the density of smooth functions in Sobolev-Orlicz spaces, Zap. Nauchn. Sem. S.-Peterburg. Otdel. Mat. Inst. Steklov. (POMI) 310 (2004), Kraev. Zadachi Mat. Fiz. i Smezh. Vopr. Teor. Funkts. 35 [34], 67-81, 226 (in Russian); English transl.: J. Math. Sci. (N.Y.) 132 (2006), 285-294.

[41] W. P. Ziemer, Weakly Differentiable Functions, Grad. Texts in Math. 120, Springer, Berlin, 1989.

Section of Applied Mathematics

Freiburg University

Eckerstrasse 1

79104 Freiburg/Breisgau, Germany

E-mail: diening@mathematik.uni-freiburg.de
Department of Mathematical Sciences

P.O. Box 3000

FI-90014 University of Oulu, Finland

E-mail: peter.hasto@helsinki.fi http://cc.oulu.fi/ phasto/

http://www.mathematik.uni-freiburg.de/IAM/homepages/diening/

Received July 1, 2006

Revised version July 31, 2007 